\title{
La crisis europea de la política: pluralismo etno-religioso y el auge del populismo y la extrema derecha en Europa
}

\author{
Cristina Astier \\ mcastier.m@gmail.com \\ Ander Errasti \\ ander.errasti.lopez@gmail.com
}

Globernance Researchers

doi: http://dx.doi.org/10.18543/ced-59-2018pp27-34

\begin{abstract}
Resumen: No genera mucha polémica sostener que la crisis económica del 2008 puede entenderse como un fracaso de los estados de bienestar europeos. Tanto el incremento de las desigualdades económicas parcialmente motivadas por esta crisis global, como el aumento de los flujos migratorios, han contribuido a crear un caldo de cultivo para el auge de los discursos de odio racial, religioso e ideológico en occidente. Sin embargo, haciendo la comparación con periodos históricos previos en los que surgieron tensiones equiparables, la ciudadanía de hoy en día puede canalizar sus intereses, necesidades, emociones, pensamientos e ideales políticos a través de las instituciones que conforman la estructura básica del estado. Así, la ciudadanía puede empoderarse canalizando sus reivindicaciones a través de mecanismos democráticos y distintas formas de participación política. Como ejemplo, una articulación relevante han sido las nuevas expresiones de populismo radical, nativismo e ideologías de extrema derecha que han surgido en varios niveles de la esfera pública y en distintas sociedades europeas. Esta combinación ha hecho que lo que era en un principio una crisis económica y de refugiados se haya convertido en una crisis que es sobre todo política.
\end{abstract}

Palabras clave: integración europea, crisis política, retos democráticos, auge de radicalismos, unidos en la diversidad.

No resulta controvertido afirmar que Europa se enfrenta a un periodo crítico: el resultado de la crisis económica del 2008 ha supuesto un reto que trasciende la dimensión meramente económica. En el plano institucional, las democracias tanto de los estados miembros como de la Unión Europea no logran abordar debidamente estas consecuencias indeseadas. El temor creciente, la sensación de inseguridad tanto dentro como entre los países, y 
las incertidumbres económicas y sociales resultantes de la crisis están cambiando el panorama político a lo largo y ancho de Europa. Se podría afirmar que la distribución injusta de los costos económicos y de otra índole generados por esta crisis, ha empujado a la ciudadanía de varios países europeos a romper con los valores fundacionales de la Unión y las fuentes tradicionales de cohesión social. Los partidos políticos al uso, los medios, el saber experto, o las instituciones públicas domésticas, europeas e internacionales han experimentado un cambio sustancial en estos últimos años como resultado de la crisis.

Estos fenómenos occidentales han coincidido con las consecuencias tanto de la denominada Primavera Árabe como de la segunda Guerra de Irak que han derivado, por lo general, en situaciones de inestabilidad política y, en última instancia, en múltiples conflictos militares en las regiones colindantes con los países de la Unión Europea. En el caso concreto de la guerra civil siria, que comenzó en el año 2011, un número creciente de refugiados que huían del conflicto se sumaron a las personas que migraron a Europa por motivos económicos desde regiones del Norte de África. En la actualidad, se estima que el número total de refugiados sirios es de alrededor de 11 millones de personas desde el comienzo de la guerra en 2011. A éstos se le suman 13,5 millones de sirios que tienen la necesidad de asistencia humanitaria dentro del país, incluyendo aquellos que sobreviven en zonas de difícil acceso. Entre estas personas, en torno a un millón han solicitado asilo en Europa. A pesar de que los estados miembros contrajeron el compromiso de garantizar asilo en la Unión Europea a un número concreto de personas, ninguno de estos Estados europeos ni las instituciones propias de la Unión Europea supieron dar una respuesta adecuada al impacto de la popularmente conocida como «crisis de los refugiados». En ese sentido, y en particular en el caso de las personas refugiadas de la guerra de siria que representan un reto urgente de integración en las sociedades europeas, la respuesta efectiva tanto de los estados miembros como de las instituciones de la Unión ha sido tanto insuficiente como poco efectiva.

En este contexto, tanto el incremento de las desigualdades económicas tras la crisis económica del 2008, que puede entenderse como un fracaso de los estados de bienestar europeos, como el incremento de los flujos migrantes han contribuido a crear un caldo de cultivo para el auge de los discursos de odio racial, religioso e ideológico en occidente. En este caso, el elemento distintivo de este periodo, comparado con periodos previos en los que surgieron tensiones equiparables, es que la ciudadanía puede recurrir a instrumentos políticos para canalizar sus intereses, necesidades, emociones, pensamientos e ideales políticos. La ciudadanía puede empoderarse canalizando sus reivindicaciones a través de meca- 
nismos democráticos y distintas formas de participación política. Como ejemplo, una articulación relevante han sido las nuevas expresiones de populismo radical, nativismo e ideologías de extrema derecha que han surgido en varios niveles de la esfera pública europea y en distintas sociedades europeas. Esta combinación ha hecho que lo que era en un principio una crisis económica y de refugiados se haya convertido en una crisis que es, también y, sobre todo, política.

Este nuevo número de los CED (Cuadernos Europeos de Deusto) es el resultado del Seminario Internacional sobre la Crisis Europea de la Política: Pluralismo Etno-religioso y el Auge del Populismo Radical, Nativismo y Extrema Derecha en Europa, organizado por el Instituto de Gobernanza Democrática (Globernance) el 16 de junio del 2017 en el Museo San Telmo de Donostia-San Sebastián. Al hilo del objetivo del evento, esta publicación pretende abordar y desgranar algunas de las dimensiones clave de este fenómeno global. Analiza, en un primer bloque de artículos de Carmen Innerarity y Sergio García, el significado exacto del Pluralismo Etno-religioso en Europa y los principios que deberían guiar la manera en la que las instituciones europeas abordan esta problemática. El volumen continúa con artículos de Beatriz Acha y Dídac Gutiérrez-Peris, centrados en las reacciones socio-políticas radicalizadas vis-á-vis esta transformación. Los artículos analizan, por una parte, cómo estos movimientos sociopolíticos conectan con los electorados y por otra parte, cómo una emoción como el miedo, genera un impacto nada desdeñable en las decisiones políticas. El volumen concluye con un artículo de Joxerramon Bengoetxea que aborda cómo estos elementos de la crisis europea de la política suponen un reto inalienable para el proceso de integración europea y el mantenimiento de los valores fundacionales europeos tales como la solidaridad y la democracia constitucional liberal.

El número comienza con un prefacio de Alexander Görlach, profesor afiliado del programa «En Defensa de la Democracia» de la F.D. Roosevelt Foundation del Adam Hous en el Harvard University College. A partir de su visión y experiencia global de la democracia, el profesor Görlach ofrece una breve revisión en profundidad de los principales retos que las fuerzas políticas iliberales y contra-cosmopolitas están suponiendo para la democracia europea.

Seguidamente, este número de los CED introduce un artículo de Carmen Innerarity, Profesora Titular de Sociología en la Universidad Pública de Navarra, titulado Secularismo Iliberal: Simulando la Inclusión Mediante la Neutralidad. El artículo analiza el modo en que, a pesar de su vocación universal e inclusiva, el secularismo se ha convertido en el principal argumento para la exclusión de prácticas religiosas musulmanas con la consiguiente limitación de las libertades individuales. Así, una institución conce- 
bida como un procedimiento para garantizar la igualdad de los ciudadanos mediante la diferenciación de las esferas política y religiosa, se vuelve una característica que define la identidad europea. En este escenario, las instituciones encargadas de garantizar la igualdad de los ciudadanos deben explicarse frente a los reclamos de acomodación planteados por los inmigrantes musulmanes. Si bien todo indica que no es posible establecer una relación causa-efecto, el clima de hostilidad hacia la diferencia cultural que se abre paso con el auge de partidos de extrema derecha en algunos países europeos, con una hostilidad clara hacia el Islam y los musulmanes, podría estar influenciando, hasta cierto punto, la orientación política de algunos gobiernos europeos en esta materia.

En línea con esta primera reflexión, el número incluye una revisión del proceso de secularización social presentada por Sergio García, profesorinvestigador en la Universidad Camilo José Cela y el CEDEU / Rey Juan Carlos, titulado Secularazión, Liberalismo y el Rol Problemático de la Religión en las Sociedades Modernas. Este artículo sostiene que hay una noción defectuosa del proceso de secularización social y que la tradición liberal da por supuesto varios conceptos problemáticos que sutilmente determinan la lógica del debate sobre la religión en las sociedades modernas. Si bien estos dos elementos no son considerados como factores causales para el auge de los movimientos populistas de extrema derecha, sí establecen el marco dentro del que se dan los debates sobre el pluralismo etno-religioso y su relación con la política. Estas dos cuestiones irresueltas dificultan la comprensión de la complejidad de los fenómenos sociales vinculados a la religión en las sociedades occidentales contemporáneas, tales como el fundamentalismo, el terrorismo, el Islam político o las reivindicaciones de otros grupos religiosos de poder participar en la esfera pública. A fin de alcanzar esos objetivos, el artículo se centra en cuatro puntos interconectados. El primero revisa, brevemente, la teoría de la secularización y el secularismo como ideología. El segundo constituye un cuestionamiento del marco liberal. El tercero plantea una vía particular de aproximarse a los fenómenos sociales vinculados con la religión. El artículo concluye examinando los factores vinculados a la religión que han alimentado a los movimientos populistas de extrema-derecha.

El segundo bloque de artículos comienza presentando el artículo de Beatriz Acha, profesora de sociología en la Universidad Pública de Navarra, titulado La Extrema Derecha en la Europa Occidental: «de Ser Marginal a Ser Popular» y ¿Vuelta a Ser Marginal? Este artículo analiza el auge de la Extrema Derecha en Europa occidental y la creciente preocupación política, social y académica ante los recientes resultados electorales de partidos políticos radicales de extrema-derecha. El artículo parte de que, desde finales de los ochenta, estamos ante una nueva (tercera) «ola» 
de radicalismo de extrema-derecha en diversos países europeos - con algunos partidos habiendo entrado en la lógica electoral y habiéndose consolidado políticamente-, que sigue la estela de múltiples análisis que ya entonces comenzaron a aproximarse a la cuestión de su popularización o aceptación «mainstream». El artículo incluye algunos datos de la evolución política y electoral de la Extrema Derecha que parecen confirmar que ese proceso de popularización efectivamente se materializó entre la década de los ochenta y los dos mil. En segundo lugar, el artículo introduce la característica principal de la llamada última fase de la evolución política de la Extrema Derecha: la cuestión de la inmigración - tema central en la Extrema Derecha - en relación con la «crisis de los refugiados», que ha acelerado la radicalización de muchos (si no todos) los partidos de Extrema Derecha (asî como algunos partidos que no eran considerados radicales). En tercer lugar, el artículo aborda ese proceso de supuesta radicalización de la Extrema Derecha. Finalmente, la conclusión especula sobre la futura evolución de la Extrema Derecha y plantea posibles vías para la investigación ulterior del fenómeno.

El segundo artículo de este bloque, Nuevo Realineamiento del Miedo en Francia y en Europa, está vinculado a esta misma temática, pero se centra en uno de los factores centrales que explican este fenómeno político: la creciente relevancia e impacto del miedo en la política europea y sus consecuencias para la democracia. Dídac Gutiérrez-Péris, profesor de la $\mathrm{Pu}-$ blic Affairs School en Sciencies Po París y Director de Investigación en Viavoice, basa su artículo principalmente en un estudio realizado a lo largo del 2016 en Francia, Polonia, el Reino Unido, España, Suecia y Alemania con el think tank Demos y el Institute Notre Europe - Institut Jacques Delors, denominado «Nothing to Fear but Fear Itself». El artículo se centra, sobre todo, en el caso de Francia y parte de la afirmación de que estamos asistiendo a una suerte de retroceso contra la aproximación 'multicultural' a la integración. Esta hipotética tendencia, como se plantea a lo largo del artículo, no siempre puede vincularse a algunos de los nuevos temores a los que las sociedades europeas se están enfrentando. Sin embargo, es el punto de partida contextual necesario para analizar uno de esos temores: el endurecimiento de las actitudes sociales en relación a la inmigración y la diversidad. El artículo identifica tres de los síntomas más comunes respecto al nuevo alineamiento del miedo en Europa. En este sentido, sugiere que Europa no sólo está asistiendo a un incremento de actitudes temerosas hacia el terrorismo o la incertidumbre, sino también a una sociedad que comienza a mostrar signos de fatiga respecto de los valores que hasta ahora habían generado la mentalidad abierta europea. Los tres síntomas identificados que describe el artículo son: i) un apoyo creciente a partidos populistas autoritarios, ii) una confianza baja y decreciente en las instituciones políticas, y 
iii) el endurecimiento de las actitudes sociales en relación a la inmigración y la diversidad. Como se muestra a lo largo del artículo, esto no siempre está vinculado a algunos de los nuevos temores que las sociedades europeas están enfrentando, si bien es el punto de partida contextual necesario para analizar uno de esos nuevos temores: el rechazo creciente hacia la migración y la diversidad.

Finalmente, Joxerramon Bengoetxea, Profesor en la Universidad del País Vasco/Euskal Herriko Unibertsitatea (UPV/EHU), plantea una reflexión general titulada ¿Una Crisis Existencial? Libertad, Tolerancia, Solidaridad, Paz; o por qué Europa es Valiosa. Este artículo aborda la crisis existencia europea. Lo hace sugiriendo que, sin desatender la relevancia del diseño institucional, la esencia del proyecto de integración europea son las personas y los pueblos, en vez de los estados. Partiendo de esa premisa, el artículo aborda dos discursos relevantes y representativos sobre la crisis existencial europea. Seguidamente trata la cuestión de la diversidad en tanto que el lema del fallido tratado constitucional europeo fue, precisamente, «unidos en la diversidad». Este reto da lugar a la necesidad de explicar la centralidad del individuo en la razón práctica, así como la importancia de los sistemas normativos. La centralidad del individuo, vinculada al valor de la libertad, se sitúa entonces en un contexto de pluralidad y diversidad. En este contexto se aborda la cuestión del auge de los movimientos reaccionarios partiendo del mapa de la pluralidad característica de Europa; el llamado multiculturalismo o pluralismo etno-religioso. El artículo concluye sugiriendo una versión del cosmopolitismo, el pluralismo hermenéutico, como una posición normativa para tratar el equilibrio entre la libertad individual y la solidaridad entre las «personas»y los «pueblos».

Como último apunte, nos gustaría agradecer a los participantes en el Seminario Internacional sobre la Crisis Europea de la Política: Pluralismo Etno-religioso y el Auge del Populismo Radical, Nativismo y Extrema Derecha en Europa, organizado por el Instituto de Gobernanza Democrática (Globernance) el 16 de junio del 2017 en el Museo San Telmo de Donostia-San Sebastián. Esta publicación no hubiera sido posible sin sus contribuciones, comentarios y fructíferos debates. En particular, querríamos reconocer la coordinación de Daniel Innerarity y el inestimable apoyo de Mikel Cabello. Asimismo, Sandra Kingery ha hecho un magnífico trabajo de revisión linguiística de los textos. Finalmente, queremos expresar nuestra gratitud a todo el equipo a cargo de Cuadernos Europeos de Deusto, con especial mención a la profesora Beatriz Pérez de las Heras y su firme convicción en este proyecto. Cualquier virtud de este número es el resultado de la combinación de todas estas contribuciones; cualquier carencia es meramente atribuible a nuestros errores. 


\section{Sobre los autores}

Ander Errasti es Doctor en Humanidades -Ética y Filosofía Política - en la Universitat Pompeu Fabra (UPF), supervisado por los doctores Sonia Arribas y Daniel Innerarity. Su investigación se centró en un análisis normativo del Cosmopolitismo de Ulrich Beck (Nations and Nationalism in a Cosmopolitanized World: Some Lessons from Ulrich Beck's Work). Fue estudiante de doctorado visitante en el Departamento de Políticas y Relaciones Internacionales de la Universidad de Oxford. Licenciado en Filosofía por la Universitat de Barcelona (UB), MA in Political Philosophy por la UPF, Diplomado en Ciencias Empresariales por la UB, Máster Avanzado en Ciencias Jurídicas por la UPF. Ha trabajado en el Instituto de Gobernanza Democrática de Donostia-San Sebastián (Globernance) desde el 2013, donde ha organizado diversos congresos internacionales, publicaciones y actividades de investigación. En la actualidad está desarrollando un proyecto de investigación en la Universidad de Edimburgo (supervisado por los profesores Michael Keating y Nicola McEwan) sobre el impacto del Brexit y el referéndum de independencia del 2014 en las instituciones de autogobierno escocesas. Finalmente, también es miembro del grupo GISME de la UB desde el 2010, dirigido por el profesor Javier Tejada. Como miembro ha organizado diversas actividades de difusión científica, incluyendo colaboraciones con el Museo del Prado, la Fundación Española de Ciencia y Tecnología o Jakiunde (la Academia Vasca de Ciencia y Humanidades). Es asistente de la Coordinación del proyecto «CCentre: hacia un envejecimiento Activo y Bienestar centrados en la ciudadanía» (2016-2019), financiado por el EIT Health y coordinado por la profesora Elena Lauroba. Fue el Project Manager del proyecto SIforAGE: Innovación Social para un Envejecimiento Activo y Saludable para un desarrollo económico sostenible (2013-2016) financiado por la Comisión Europea.

Cristina Astier es estudiante de doctorado en la especialidad de Filosofía Política en la Universitat Pompeu Fabra (Barcelona, UPF), investigadora de Globernance (Instituto de Gobernanza Democrática de San Sebastián) e investigadora de GISME (Grupo de Investigación en Soluciones Matemáticas para Entidades) un grupo multidisciplinar de la Universitat de Barcelona. Licenciada en Filosofía por la Universitat de Barcelona y Máster en Filosofía Política por la UPF. Ha sido estudiante de doctorado visitante en el Departamento de Políticas y Relaciones Internacionales, y del Centro de Estudios de Justicia Social de la Universidad de Oxford. Su investigación está centrada en cuestiones de ética global, justicia distributiva global, justicia en el comercio internacional y consumo, y la legitimidad de 
las instituciones de gobernanza globales. Combina su actividad académica con actividades profesionales no académicas, especialmente en proyectos europeos. Desde 2012 hasta 2016 fue asistente de la coordinación y responsable del equipo de comunicación del proyecto europeo SIforAGE (Innovación Social para un Envejecimiento Activo y Saludable) financiado bajo el Séptimo Programa Marco de la Comisión Europea. Desde el 2016 es la gestora de contenidos y comunicación del proyecto europeo CCentre. El proyecto está financiado por el EIT Health (Instituto Europeo de Innovación y Tecnología), un órgano de la Unión Europea. 


\section{Derechos de autor}

Los derechos de autor (para la distribución, comunicación pública, reproducción e inclusión en bases de datos de indexación y repositorios institucionales) de esta publicación (Cuadernos Europeos de Deusto, CED) pertenecen a la editorial Universidad de Deusto. El acceso al contenido digital de cualquier número de Cuadernos Europeos de Deusto es gratuito inmediatamente después de su publicación. Los trabajos podrán leerse, descargarse, copiar y difundir en cualquier medio sin fines comerciales y según lo previsto por la ley; sin la previa autorización de la Editorial (Universidad de Deusto) o el autor. Así mismo, los trabajos editados en CED pueden ser publicados con posterioridad en otros medios o revistas, siempre que el autor indique con claridad y en la primera nota a pie de página que el trabajo se publicó por primera vez en $C E D$, con indicación del número, año, páginas y DOI (si procede). Cualquier otro uso de su contenido en cualquier medio o formato, ahora conocido o desarrollado en el futuro, requiere el permiso previo por escrito del titular de los derechos de autor.

\section{Copyright}

Copyright (for distribution, public communication, reproduction and inclusion in indexation databases and institutional repositories) of this publication (Cuadernos Europeos de Deusto, CED) belongs to the publisher University of Deusto. Access to the digital content of any Issue of Cuadernos Europeos de Deusto is free upon its publication. The content can be read, downloaded, copied, and distributed freely in any medium only for non-commercial purposes and in accordance with any applicable copyright legislation, without prior permission from the copyright holder (University of Deusto) or the author. Thus, the content of $C E D$ can be subsequently published in other media or journals, as long as the author clearly indicates in the first footnote that the work was published in $C E D$ for the first time, indicating the Issue number, year, pages, and DOI (if applicable). Any other use of its content in any medium or format, now known or developed in the future, requires prior written permission of the copyright holder. 